\title{
H-INFINITY FILTERING FOR A CLASS OF STOCHASTIC BILINEAR SYSTEMS WITH MULTIPLICATIVE NOISE
}

\author{
S. Halabi, H. Rafaralahy, H. Souley Ali and M. Zasadzinski
}

\author{
Université Henri Poincaré - Nancy I, \\ CRAN UMR 7039 - CNRS \\ IUT de Longwy, \\ 186, rue de Lorraine, 54400 Cosnes et Romain, FRANCE \\ e-mail : \{souley,mzasad\}@iut-longwy.uhp-nancy.fr
}

\begin{abstract}
In this paper, the purpose is to design a filter for a stochastic bilinear system which satisfies an $\mathcal{H}_{\infty}$ prescribed norm constraint and such that the estimation error is mean-square stable. The system under consideration is bilinear in control input and is subjected to multiplicative noise in both the state and the measurement equations. The system is also corrupted by deterministic perturbations. The proposed approach is based on the resolution of LMI and the filter design requires to satisfy a rank condition. Copyright (C)2005 IFAC
\end{abstract}

Keywords: Filtering, Stochastic systems, Itô equation, Lyapunov stochastic function, LMI.

\section{INTRODUCTION}

The bilinear system is sometimes a good mean to represent physical systems when the linear representation is not sufficiently significant. The stochastic systems get a great importance in the last decades as shown by numerous references (Has'minskii, 1980; Kozin, 1969; Florchinger, 1997; Mao, 1997; Carravetta et al., 2000; Germani et al., 2002; Xu and Chen, 2003).

Generally, the term of bilinear stochastic system designs a system with multiplicative noise instead of additive one (Carravetta et al., 2000; Germani et al., 2002). The $\mathcal{H}_{\infty}$ filtering for systems with multiplicative noise has been treated in many papers (Gershon et al., 2001; Xu and Chen, 2002; Stoica, 2002). In (Stoica, 2002), the problem of reduced-order $\mathcal{H}_{\infty}$ filtering for a class of stochastic systems is solved in terms of two LMIs conditions coupled by a rank condition. The considered system is deterministic while the measurements are subjected to a multiplicative noise. In $\left(\mathrm{Xu}\right.$ and Chen, 2002), the reduced-order $\mathcal{H}_{\infty}$ filtering for stochastic systems with multiplicative noise and corrupted by deterministic input disturbance is treated. Notice that the measurement equation in this paper is not corrupted by noise, the problem is resolved in term of two LMIs and a coupling non convex rank constraint set. The $\mathcal{H}_{\infty}$ filtering with noisy measurement equation is considered in (Gershon et al., 2001). The dynamic output feedback for stochastic system subjected to both deterministic and stochastic perturbations is solved in (Hinrichsen and Pritchard, 1998).

In this paper, the bilinearity is also between the state and the control input. The goal is to design a filter for this kind of stochastic systems such that the estimation error system is mean-square stable and satisfies an $\mathcal{H}_{\infty}$ norm constraint. The approach is based on a change of variable on the control input to transform the problem into a robust stochastic filtering one. Then the Itô formula and the LMI method permit to obtain a condition to be verified to ensure the existence of the filter. Let $L_{2}\left(\Omega, \mathbb{R}^{k}\right)$ be the space of square-integrable $\mathbb{R}^{k}$-valued functions on the probability space $(\Omega, \mathcal{F}, \mathcal{P})$ where $\Omega$ is the sample space, $\mathcal{F}$ is a $\sigma$-algebra of subsets of the sample space called 
events and $\mathcal{P}$ is the probability measure on $\mathcal{F}$. $\left(\mathcal{F}_{t}\right)_{t \geqslant 0}$ denote an increasing family of $\sigma$-algebras $\left(\mathcal{F}_{t}\right) \in \mathcal{F}$. We also denote by $\widehat{L}_{2}\left([0, \infty) ; \mathbb{R}^{k}\right)$ the space of non-anticipatory square-integrable stochastic process $f()=.(f(t))_{t \in[0, \infty)}$ in $\mathbb{R}^{k}$ with respect to $\left(\mathcal{F}_{t}\right)_{t \in[0, \infty)}$ satisfying

$$
\|f\|_{\widehat{L}_{2}}^{2}=\mathbf{E}\left\{\int_{0}^{\infty}\|f(t)\|^{2} \mathrm{~d} t\right\}<\infty
$$

where $\|$.$\| is the well-known Euclidean norm.$

\section{PROBLEM STATEMENT}

Let us consider the following Itô stochastic bilinear system (Has'minskii, 1980; Florchinger, 1997)

$$
\left\{\begin{aligned}
\mathrm{d} x(t) & =(A x(t)+u(t) B x(t)) \mathrm{d} t \\
& +F x(t) \mathrm{d} w(t)+G v(t) \mathrm{d} t \\
\mathrm{~d} y(t) & =C x(t) \mathrm{d} t+H x(t) \mathrm{d} w \\
z(t) & =L x(t)
\end{aligned}\right.
$$

where $x(t) \in \mathbb{R}^{n}$ is the state vector, $y(t) \in \mathbb{R}^{p}$ is the output, $u(t) \in \mathbb{R}$ is the control input, $v(t) \in \mathbb{R}^{r}$ is the disturbance vector and $z(t) \in \mathbb{R}^{q}$ is the state linear combination to be estimated. $w(t)$ is a zero mean scalar Wiener process verifying (Has'minskii, 1980)

$$
\mathbf{E}(\mathrm{d} w(t))=0 \text { and } \mathbf{E}\left(\mathrm{d} w(t)^{2}\right)=\mathrm{d} t .
$$

To simplify the notation and without loss of generality, we consider only the single control input case. As in the most cases for physical processes, assume that the stochastic bilinear system (1) has known bounded control input, i.e. $u(t) \in \Gamma \subset \mathbb{R}$, where

$$
\Gamma:=\left\{u(t) \in \mathbb{R} \mid u_{\min } \leqslant u(t) \leqslant u_{\max }\right\} .
$$

Assumption 1. We suppose that $v(t) \in \widehat{L}_{2}^{2}$.

Definition 2. (Hinrichsen and Pritchard, 1998; Ryashko and Schurtz, 1996) The stochastic system (1) with $v(t) \equiv 0$ is asymptotically meansquare stable if all initial states $x(0)$, yield

$$
\lim _{t \rightarrow \infty} \mathbf{E}\|x(t)\|^{2}=0, \quad \forall u(t) \in \Gamma .
$$

Assumption 3. The stochastic bilinear system (1) with $v(t)=0$ is assumed to be asymptotically mean-square stable.

In this paper, the aim is to design a filter in the following form

$$
\left\{\begin{aligned}
\mathrm{d} \hat{x}(t)= & (A \hat{x}(t)+u(t) B \hat{x}(t)) \mathrm{d} t \\
& +K(\mathrm{~d} y(t)-C \hat{x}(t) \mathrm{d} t) \\
& +u(t) \bar{K}(\mathrm{~d} y(t)-C \hat{x}(t) \mathrm{d} t) \\
\hat{z}(t)= & L \hat{x}(t)
\end{aligned}\right.
$$

with $K$ and $\bar{K}$ are the gains to design in order to ensure that the estimation error $x-\hat{x}$ is meansquare stable. Notice that the estimation error $x(t)-\hat{x}(t)$ has the following dynamics

$$
\begin{aligned}
& \mathrm{d} e(t)=\mathrm{d} x(t)-\mathrm{d} \hat{x}(t) \\
& \quad=(A-K C+u(t)(B-\bar{K} C)) e(t) \mathrm{d} t \\
& +(F-K H-u(t) \bar{K} H) x(t) \mathrm{d} w+G v(t) \mathrm{d} t .
\end{aligned}
$$

Let us consider the following augmented state vector

$$
\xi^{T}(t)=\left[x^{T}(t) e^{T}(t)\right] .
$$

Then the dynamics of the augmented system is given by

$$
\left\{\begin{aligned}
\mathrm{d} \xi(t)= & (\mathcal{A}+u(t) \mathcal{B}) \xi(t) \mathrm{d} t \\
& +\left(\mathcal{A}_{0}+u(t) \mathcal{B}_{0}\right) \xi(t) \mathrm{d} w(t)+\widetilde{G} v(t) \mathrm{d} t \\
\tilde{z}(t)= & z(t)-\hat{z}(t)=\widetilde{L} \xi
\end{aligned}\right.
$$

with

$$
\begin{aligned}
\mathcal{A} & =\left[\begin{array}{cc}
A & 0 \\
0 & A-K C
\end{array}\right], \mathcal{B}=\left[\begin{array}{cc}
B & 0 \\
0 & B-\bar{K} C
\end{array}\right], \widetilde{G}=\left[\begin{array}{l}
G \\
G
\end{array}\right] \\
\mathcal{A}_{0} & =\left[\begin{array}{cr}
F & 0 \\
F-K H & 0
\end{array}\right], \mathcal{B}_{0}=\left[\begin{array}{cc}
0 & 0 \\
-\bar{K} H & 0
\end{array}\right], \widetilde{L}=\left[\begin{array}{ll}
0 & L
\end{array}\right] .
\end{aligned}
$$

Now, we introduce the two following definition.

Definition 4. (Hinrichsen and Pritchard, 1998; $\mathrm{Xu}$ and Chen, 2003) The stochastic system (8) is said to be externally stable if, for every $v(t) \in \widehat{L}_{2}\left([0, \infty] ; \mathbb{R}^{m}\right), \exists \gamma>0$ such that $\tilde{z}(t)=$ $z(t)-\hat{z}(t)$ is mean-square stable and the following $\mathcal{H}_{\infty}$ performance

$$
\|\tilde{z}(t)\|_{\widehat{L}_{2}}^{2} \leqslant \gamma\|v(t)\|_{\widehat{L}_{2}}^{2}
$$

holds.

Problem 5. Given a real $\gamma>0$, the goal is to design an asymptotically stable filter of the form of (5) such that the augmented system (8) is asymptotically mean-square stable and the following $\mathcal{H}_{\infty}$ performance

$$
\|\tilde{z}(t)\|_{\widehat{L}_{2}}^{2} \leqslant \gamma\|v\|_{\widehat{L}_{2}}^{2}
$$

is achieved.

\section{ANALYSIS OF THE STABILITY OF THE AUGMENTED SYSTEM}

In this part, we study the conditions which ensure the mean-square stability of system (8) i.e. with $v(t)=0$. The gains $K$ and $\bar{K}$ are considered to be known in order to avoid bilinearity in the derivation of the stability condition.

For this purpose, consider the following Lyapunov function

$$
V(\xi)=\xi^{T} \mathcal{P} \xi
$$


using the Itô's formula (Has'minskii, 1980), (Florchinger, 1997; Kozin, 1969; Mao, 1997; Xu and Chen, 2003) we have

$\mathrm{d} V(\xi(t))=L V(\xi(t)) \mathrm{d} t+2 \xi^{T}(t) \mathcal{P}\left(\mathcal{A}_{0}+u(t) \mathcal{B}_{0}\right) \xi(t) \mathrm{d} w(t)$

with

$$
\begin{aligned}
& L V(\xi(t))=2 \xi^{T}(t) \mathcal{P}(\mathcal{A}+u(t) \mathcal{B}) \xi(t)+ \\
& \quad \xi^{T}(t)\left(\mathcal{A}_{0}+u(t) \mathcal{B}_{0}\right)^{T} \mathcal{P}\left(\mathcal{A}_{0}+u(t) \mathcal{B}_{0}\right) \xi(t) .
\end{aligned}
$$

Then relation (13) is rewritten as

$$
\begin{gathered}
\mathrm{d} V(\xi(t))=\xi^{T}(t)\left\{\mathcal{P} \mathcal{A}+\mathcal{A}^{T} \mathcal{P}+u(t)\left(\mathcal{B}^{T} \mathcal{P}+\mathcal{P} \mathcal{B}\right)\right. \\
\left.+\left(\mathcal{A}_{0}+u(t) \mathcal{B}_{0}\right)^{T} \mathcal{P}\left(\mathcal{A}_{0}+u(t) \mathcal{B}_{0}\right)\right\} \xi(t) \mathrm{d} t \\
+2 \xi^{T}(t) \mathcal{P}\left(\mathcal{A}_{0}+u(t) \mathcal{B}_{0}\right) \xi(t) \mathrm{d} w(t)
\end{gathered}
$$

To study the stability of this system, we introduce a change of variable on the control input $u(t)$ in order to reduce the conservatism introduced by the assumption that $u(t)$ is bounded (see (3)). Let

$$
u(t)=\alpha+\sigma \varepsilon(t)
$$

where $\alpha \in \mathbb{R}$ and $\sigma \in \mathbb{R}$ are given by

$$
\alpha=\frac{1}{2}\left(u_{\min }+u_{\max }\right), \sigma=\frac{1}{2}\left(u_{\max }-u_{\min }\right) .
$$

The new "uncertain" variable is $\varepsilon(t) \in \bar{\Gamma} \subset \mathbb{R}$ where the polytope $\bar{\Gamma}$ is defined by

$$
\bar{\Gamma}:=\left\{\varepsilon(t) \in \mathbb{R} \mid \varepsilon_{\min }=-1 \leqslant \varepsilon(t) \leqslant \varepsilon_{\max }=1\right\} .
$$

Equation (15) is rewritten as

$$
\begin{aligned}
& \mathrm{d} V(\xi(t))= \\
& \quad \xi^{T}(t)\left\{\mathcal{P} \mathcal{A}_{t}+\mathcal{A}_{t}^{T} \mathcal{P}+\mathcal{P} \Delta \mathcal{A}(t)+\Delta \mathcal{A}(t)^{T} \mathcal{P}\right. \\
& \left.+\left(\mathcal{A}_{0 t}+\Delta \mathcal{A}_{0 t}(t)\right)^{T} \mathcal{P}\left(\mathcal{A}_{0 t}+\Delta \mathcal{A}_{0 t}(t)\right)\right\} \xi(t) \mathrm{d} t \\
& \quad+2 \xi^{T}(t) \mathcal{P}\left(\mathcal{A}_{0 t}+\Delta \mathcal{A}_{0 t}(t)\right) \xi(t) \mathrm{d} w(t)
\end{aligned}
$$

for the system (see (8))

$$
\left\{\begin{aligned}
\mathrm{d} \xi(t)= & \left(\mathcal{A}_{t}+\Delta \mathcal{A}(t) \xi(t) \mathrm{d} t+\widetilde{G} v(t) \mathrm{d} t\right. \\
& +\left(\mathcal{A}_{0 t}+\Delta \mathcal{A}_{0 t}(t)\right) \xi(t) \mathrm{d} w(t) \\
\tilde{z}(t)= & \widetilde{L} \xi
\end{aligned}\right.
$$

where

$$
\begin{aligned}
\mathcal{A}_{t} & =(\mathcal{A}+\alpha \mathcal{B}), \Delta \mathcal{A}(t)=H_{1} \Delta_{\xi}(\varepsilon(t)) H_{2}, \\
\mathcal{A}_{0 t} & =\left(\mathcal{A}_{0}+\alpha \mathcal{B}_{0}\right), \Delta \mathcal{A}_{0 t}(t)=H_{10} \Delta_{\xi}(\varepsilon(t)) H_{2}
\end{aligned}
$$

with

$$
H_{1}=\sigma \mathcal{B}, H_{10}=\sigma \mathcal{B}_{0}, \Delta_{\xi}(\varepsilon(t))=\varepsilon(t) \text { and } H_{2}=I_{2 n} \text {. }
$$

From the majoration lemma (Xu and Chen, 2003), we have (with $\epsilon_{1}>0$ and $\epsilon_{2}>0$ )

$$
\begin{aligned}
& 2 \xi^{T}(t) \mathcal{P} \Delta \mathcal{A}(t) \xi(t) \leqslant \\
& \xi^{T}(t)\left[\epsilon_{1}+\epsilon_{1}^{-1} \mathcal{P} \sigma \mathcal{B} \mathcal{B}^{T} \sigma \mathcal{P}\right] \xi(t)
\end{aligned}
$$

and

$$
\begin{aligned}
& \left(\mathcal{A}_{0 t}+\Delta \mathcal{A}_{0 t}(t)\right)^{T} \mathcal{P}\left(\mathcal{A}_{0 t}+\Delta \mathcal{A}_{0 t}(t)\right) \leqslant \\
& \quad \mathcal{A}_{0}^{T}\left(\mathcal{P}^{-1}-\epsilon_{2}^{-1} H_{10} H_{10}^{T}\right)^{-1} \mathcal{A}_{0}+\epsilon_{2} H_{2}^{T} H_{2} .
\end{aligned}
$$

$$
\begin{aligned}
& \mathrm{d} V(\xi(t)) \leqslant \xi^{T}(t)\left\{\mathcal{P} \mathcal{A}_{t}+\mathcal{A}_{t}^{T} \mathcal{P}+\epsilon_{1} I_{2 n}\right. \\
& +\epsilon_{1}^{-1} \mathcal{P} \sigma \mathcal{B B}^{T} \sigma \mathcal{P}+\mathcal{A}_{0}^{T}\left(\mathcal{P}^{-1}-\epsilon_{2}^{-1} H_{10} H_{10}^{T}\right)^{-1} \mathcal{A}_{0} \\
& \left.\quad+\epsilon_{2} H_{2}^{T} H_{2}\right\} \xi(t) \mathrm{d} t \\
& \quad+2 \xi^{T}(t) \mathcal{P}\left(\mathcal{A}_{0 t}+\Delta \mathcal{A}_{0 t}(t)\right) \xi(t) \mathrm{d} w(t) .
\end{aligned}
$$

Then the following theorem is given to ensure the asymptotically mean-square stability of the augmented system (20).

Theorem 6. The system (20) with $v(t)=0$ is mean-square stable it there exist $\mathcal{P}=\mathcal{P}^{T}>0$ and two real $\epsilon_{1}>0, \epsilon_{2}>0$ such that the following LMI

$$
\left[\begin{array}{cccc}
(1,1) & \mathcal{P} H_{1} & \mathcal{A}_{0}^{T} \mathcal{P} & 0 \\
H_{1}^{T} \mathcal{P} & -\epsilon_{1} I_{2 n} & 0 & 0 \\
\mathcal{P} \mathcal{A}_{0} & 0 & -\mathcal{P} & \mathcal{P} H_{10} \\
0 & 0 & H_{10}^{T} \mathcal{P} & -\epsilon_{2}^{-1} I_{2 n}
\end{array}\right]<0
$$

holds, with

$$
(1,1)=\mathcal{P} \mathcal{A}_{t}+\mathcal{A}_{t}^{T} \mathcal{P}+\left(\epsilon_{1}+\epsilon_{2}\right) H_{2}^{T} H_{2} .
$$

Proof. Using the Schur lemma, theorem 6 gives

$$
\begin{aligned}
& \mathcal{P} \mathcal{A}_{t}+\mathcal{A}_{t}^{T} \mathcal{P}+\epsilon_{1} H_{2}^{T} H_{2}+\epsilon_{1}^{-1} \mathcal{P} H_{1} H_{1}^{T} \mathcal{P} \\
+ & \mathcal{A}_{0}^{T}\left(\mathcal{P}^{-1}-\epsilon_{2} H_{10} H_{10}^{T}\right)^{-1} \mathcal{A}_{0}+\epsilon_{2}^{-1} H_{2}^{T} H_{2}=-\mathcal{K}<0 .
\end{aligned}
$$

Note that from $(26) \lambda_{\min }(\mathcal{K})>0$ where $\lambda_{\min }$ is the smallest eigenvalue of $\mathcal{K}$. This and (25) yield to

$$
\begin{aligned}
& \mathrm{d} V(\xi(t)) \leqslant-\lambda_{\min }(\mathcal{K})\|\xi(t)\|^{2} \mathrm{~d} t \\
& \quad+2 \xi^{T}(t) \mathcal{P}\left(\mathcal{A}_{0 t}+\Delta \mathcal{A}_{0 t}(t)\right) \xi(t) \mathrm{d} w(t) .
\end{aligned}
$$

Let $\beta>0$ be given, using the integration-by-part formula (Mao, 1997; Xu and Chen, 2003), we can derive that

$$
\mathrm{d}\left[e^{\beta t} V(\xi(t))\right]=e^{\beta t}[\beta V(\xi(t)) \mathrm{d} t+\mathrm{d} V(\xi(t))]
$$

which can be bounded as

$$
\begin{aligned}
& \mathrm{d}\left[e^{\beta t} V(\xi(t))\right] \leqslant \\
& \quad e^{\beta t}\left(\left[-\beta \lambda_{\max }(\mathcal{P})-\lambda_{\min }(K)\right]\|\xi(t)\|^{2}\right) \mathrm{d} t \\
& +2 e^{\beta t} \xi^{T}(t) \mathcal{P}\left(\mathcal{A}_{0 t}+\Delta \mathcal{A}_{0 t}(t)\right) \xi(t) \mathrm{d} w(t) .
\end{aligned}
$$

Since

$$
\left.\left[-\beta \lambda_{\max }(\mathcal{P})-\lambda_{\min }(K)\right]\|\xi(t)\|^{2}\right) \leqslant 0,
$$

then inequalities (30) and (31) imply that

$\mathrm{d}\left[e^{\beta t} V(\xi(t))\right] \leqslant 2 e^{\beta t} \xi^{T}(t) \mathcal{P}\left(\mathcal{A}_{0 t}+\Delta \mathcal{A}_{0 t}(t)\right) \xi(t) \mathrm{d} w(t)$.

Integrating both sides from 0 to $t>0$ and then taking expectation give

$$
\begin{gathered}
\left.\left.e^{\beta t} \mathbf{E}\left[\xi^{T}(t) \mathcal{P} \xi(t)\right)\right]-e^{\beta \times 0} \mathbf{E}\left[\xi^{T}(0) \mathcal{P} \xi(0)\right)\right] \leqslant \\
\int_{0}^{t} 2 e^{\beta t} \xi^{T}(s) \mathcal{P}\left(\mathcal{A}_{0 t}+\Delta \mathcal{A}_{0 t}(s)\right) \xi(s) \mathbf{E}[\mathrm{d} w(s)] .
\end{gathered}
$$


Now using the properties (2), the right term of inequality (33) is given by

$$
\int_{0}^{t} 2 e^{\beta t} \xi^{T}(s) \mathcal{P}\left(\mathcal{A}_{0 t}+\Delta \mathcal{A}_{0 t}(s)\right) \xi(s) \mathbf{E}[\mathrm{d} w(s)]=0 .
$$

Then (33) can be rewritten as

$$
\left.e^{\beta t} \mathbf{E}\left[\xi^{T}(t) \mathcal{P} \xi(t)\right)\right] \leqslant c
$$

or equivalently

$$
\lambda_{\min }(\mathcal{P}) \mathbf{E}\|\xi(t)\|^{2} \leqslant \mathbf{E}\left[\xi^{T}(t) \mathcal{P} \xi(t)\right] \leqslant c e^{-\beta t}
$$

where $\left.c=\mathbf{E}\left[\xi^{T}(0) \mathcal{P} \xi(0)\right)\right]$ is a positive constant.

Finally, from (36) the following inequality

$$
\mathbf{E}\|\xi(t)\|^{2} \leqslant \frac{c}{\lambda_{\min }(\mathcal{P})} e^{-\beta t}
$$

ensures that the augmented system (7) is asymptotically mean-square stable.

\section{4. $\mathcal{H}_{\infty}$ PERFORMANCE}

From section 3 , the following theorem is then given for the filter synthesis

Theorem 7. The filtering problem 5 is resolved for the system (1) with the filter (5) if there exist matrices $P_{1}=P_{1}^{T}>0, P_{2}=P_{2}^{T}>0, P_{3}>0, Y_{2}$, $\bar{Y}_{2}, Y_{3}$ and $\bar{Y}_{3}$ such that the following LMIs hold

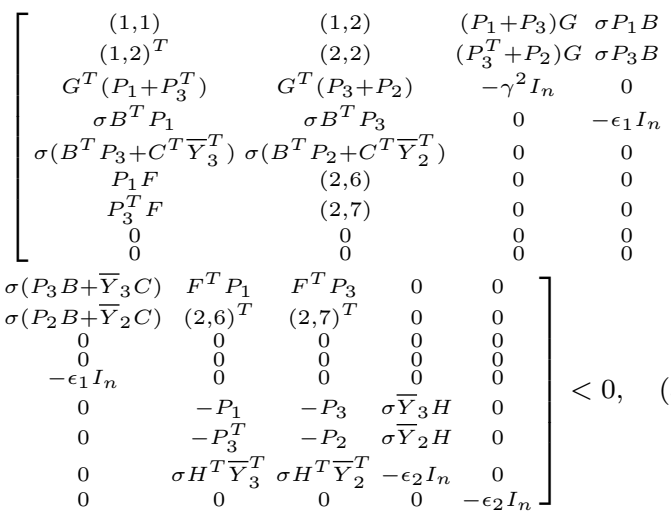

$$
\begin{aligned}
& {\left[\begin{array}{cc}
P_{1} & P_{3} \\
P_{3}^{T} & P_{2}
\end{array}\right]>0}
\end{aligned}
$$

where

$$
\begin{aligned}
(1,1)= & P_{1} A_{\alpha}+A_{\alpha}^{T} P_{1}+\left(\epsilon_{1}+\epsilon_{2}\right) I_{n}, \\
(1,2)= & P_{3} A_{\alpha}+A_{\alpha}^{T} P_{3}+Y_{3} C+\bar{Y}_{3} C_{\alpha}, \\
(2,2)= & P_{2} A_{\alpha}+A_{\alpha}^{T} P_{2}+Y_{2} C+C^{T} Y_{2}^{T}+ \\
& \bar{Y}_{2} C_{\alpha}+C_{\alpha}^{T} \bar{Y}_{2}^{T}+\left(\epsilon_{1}+\epsilon_{2}\right) I_{n}+L^{T} L, \\
(2,6)= & P_{3} F+Y_{3} H, \\
(2,7)= & P_{2} F+Y_{2} H,
\end{aligned}
$$

$A_{\alpha}=A+\alpha B, \quad C_{\alpha}=\alpha C$,

$Y_{i}=-P_{i} K$ and $\bar{Y}_{i}=-P_{i} \bar{K}$ with $i=2,3$.
Proof. Consider the Lyapunov matrix $\mathcal{P}$ given by

$$
\mathcal{P}=\left[\begin{array}{ll}
P_{1} & P_{3} \\
P_{3}^{T} & P_{2}
\end{array}\right] \text { with } P_{j} \in \mathbb{R}^{n \times n} \quad j=1, \ldots, 3 .
$$

We will use Itô formula again to the system (20) and we get

$$
\begin{aligned}
& \mathrm{d} V(\xi(t))=L V(\xi(t)) \mathrm{d} t \\
& \quad+2 \xi^{T}(t) \mathcal{P}\left(\mathcal{A}_{0 t}+\Delta \mathcal{A}_{0 t}(t)\right) \xi(t) \mathrm{d} w(t)
\end{aligned}
$$

with

$$
\begin{aligned}
& L V(\xi(t))=2 \xi^{T}(t) \mathcal{P}\left(\left(\mathcal{A}_{t}+\Delta \mathcal{A}(t)\right) \xi(t)+\widetilde{G} v(t)\right) \\
& +\xi^{T}(t)\left(\mathcal{A}_{0 t}+\Delta \mathcal{A}_{0 t}(t)\right)^{T} \mathcal{P}\left(\mathcal{A}_{0 t}+\Delta \mathcal{A}_{0 t}(t)\right) \xi(t) .
\end{aligned}
$$

Similarly to the derivation of (28), we have

$$
\begin{aligned}
\mathrm{d} V(\xi(t)) \leqslant & {\left[\begin{array}{ll}
\xi(t)^{T} & v(t)^{T}
\end{array}\right] \Theta\left[\begin{array}{l}
\xi(t) \\
v(t)
\end{array}\right] \mathrm{d} t } \\
& +2 \xi^{T}(t) \mathcal{P}\left(\mathcal{A}_{0 t}+\Delta \mathcal{A}_{0 t}(t)\right) \mathrm{d} w(t)
\end{aligned}
$$

with

$$
\begin{aligned}
\Theta= & {\left[\begin{array}{cc}
\mathcal{P} \mathcal{A}_{t}+\mathcal{A}_{t}^{T} \mathcal{P}+\epsilon_{1}^{-1} \mathcal{P} H_{1} H_{1}^{T} \mathcal{P} & \mathcal{P} \widetilde{G} \\
\widetilde{G}^{T} \mathcal{P} & 0
\end{array}\right] } \\
& +\left[\begin{array}{c}
\mathcal{A}_{0}^{T} \\
0
\end{array}\right]\left(\mathcal{P}^{-1}-\epsilon_{2}^{-1} H_{10} H_{10}^{T}\right)^{-1}\left[\begin{array}{ll}
\mathcal{A}_{0} & 0
\end{array}\right] \\
& +\epsilon_{1}\left[\begin{array}{cr}
H_{2}^{T} H_{2} & 0 \\
0 & 0
\end{array}\right]+\epsilon_{2}\left[\begin{array}{c}
H_{2}^{T} \\
0
\end{array}\right]\left[\begin{array}{ll}
H_{2} & 0
\end{array}\right] .
\end{aligned}
$$

By applying Schur we have

$$
\begin{aligned}
\Theta & =\left[\begin{array}{ccc}
\mathcal{P} \mathcal{A}_{t}+\mathcal{A}_{t}^{T} \mathcal{P} & \mathcal{P} H_{1} & \mathcal{P} \widetilde{G} \\
H_{1}^{T} \mathcal{P} & \epsilon_{1} I_{2 n} & 0 \\
\mathcal{P} \widetilde{G} & 0 & 0
\end{array}\right] \\
& +\left[\begin{array}{c}
\mathcal{A}_{0}^{T} \\
0 \\
0
\end{array}\right]\left(\mathcal{P}^{-1}-\epsilon_{2}^{-1} H_{10} H_{10}^{T}\right)^{-1}\left[\begin{array}{lll}
\mathcal{A}_{0} & 0 & 0
\end{array}\right] \\
& +\epsilon_{1}\left[\begin{array}{ccc}
H_{2}^{T} H_{2} & 0 & 0 \\
0 & 0 & 0 \\
0 & 0 & 0
\end{array}\right]+\epsilon_{2}\left[\begin{array}{c}
H_{2}^{T} \\
0 \\
0
\end{array}\right]\left[\begin{array}{lll}
H_{2} & 0 & 0
\end{array}\right] .
\end{aligned}
$$

then by applying Schur to the second term we have

$\Theta=\left[\begin{array}{ccccc}\mathcal{P} \mathcal{A}_{t}+\mathcal{A}_{t}^{T} \mathcal{P}+\left(\epsilon_{1}+\epsilon_{2}\right) H_{2}^{T} H_{2} & \mathcal{P} H_{1} & \mathcal{P} \widetilde{G} & \mathcal{A}_{0}^{T} \mathcal{P} & 0 \\ H_{1}^{T} \mathcal{P} & \epsilon_{1} I_{2 n} & 0 & 0 & 0 \\ \mathcal{P} \widetilde{G} & 0 & 0 & 0 & 0 \\ \mathcal{P} \mathcal{A}_{0} & 0 & 0 & -\mathcal{P} & \mathcal{P} H_{10} \\ 0 & 0 & 0 & H_{10}^{T} \mathcal{P} & -\epsilon_{2}^{-1} I_{2 n}\end{array}\right]$.

Integrating both sides of (45) from 0 to $t>0$ with $\Theta$ given by (46) and taking the expectation, we have

$$
\begin{aligned}
& \mathbf{E}[V(\xi(t))] \leqslant \mathbf{E}[V(\xi(0))] \\
& +\mathbf{E}\left\{\int _ { 0 } ^ { t } \left[\begin{array}{ll}
\xi(s)^{T} & \left.\left.v(s)^{T}\right] \Theta\left[\begin{array}{l}
\xi(s) \\
v(s)
\end{array}\right] \mathrm{d} s\right\} .
\end{array}\right.\right.
\end{aligned}
$$

Then, from this and by applying the Schur lemma (three times) to (38) and finally pre-multiplying 


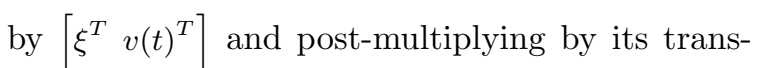
pose, we deduce the following inequality

$$
\begin{aligned}
& {\left[\begin{array}{ll}
\xi(t)^{T} & v(t)^{T}
\end{array}\right] \Theta\left[\begin{array}{l}
\xi(t) \\
v(t)
\end{array}\right]} \\
& \quad+\left[\begin{array}{ll}
\xi(t)^{T} & v(t)^{T}
\end{array}\right]\left[\begin{array}{cc}
\widetilde{L}^{T} \widetilde{L} & 0 \\
0 & -\gamma^{2} I
\end{array}\right]\left[\begin{array}{l}
\xi(t) \\
v(t)
\end{array}\right]<0,
\end{aligned}
$$

notice that the LMI (38) is equivalent to

$$
\Theta+\left[\begin{array}{cc}
\widetilde{L}^{T} \widetilde{L} & 0 \\
0 & -\gamma^{2} I
\end{array}\right]<0
$$

which ends the proof.

The gains $K$ and $\bar{K}$ are then obtained by solving the following equation

$$
\left[\begin{array}{l}
-P_{2} \\
-P_{3}
\end{array}\right]\left[\begin{array}{ll}
K & \bar{K}
\end{array}\right]=\left[\begin{array}{ll}
Y_{2} & \bar{Y}_{2} \\
Y_{3} & \bar{Y}_{3}
\end{array}\right] .
$$

Note that $K$ and $\bar{K}$ exist if and only if the following rank condition is satisfied

$$
\operatorname{rank}\left[\begin{array}{c}
P_{2} \\
P_{3}
\end{array}\right]=\operatorname{rank}\left[\begin{array}{ccc}
Y_{2} & \bar{Y}_{2} & P_{2} \\
Y_{3} & \bar{Y}_{3} & P_{3}
\end{array}\right]
$$

\section{CONCLUSION}

In this paper, a method has been proposed to resolve the problem of $\mathcal{H}_{\infty}$ filter design for bilinear stochastic system with multiplicative noise and bounded control output. An LMI approach and a rank condition are proposed for the design of the filter to ensure an $\mathcal{H}_{\infty}$ disturbance attenuation.

\section{REFERENCES}

Carravetta, F., A. Germani and M.K. Shuakayev (2000). A new suboptimal approach to the filtering problem for bilinear stochastic differential systems. SIAM J. Contr. Opt. 38, 11711203.

Florchinger, P. (1997). Feedback stabilization of affine in the control stochastic differential systems by the control lyapunov function method. SIAM J. Contr. Opt. 35, 500-511.

Germani, A., C. Manes and P. Palumbo (2002). Linear filtering for bilinear stochastic differential systems with unknown inputs. IEEE Trans. Aut. Contr. 47, 1726-1730.

Gershon, E., D.J.N. Limebeer, U. Shaked and I. Yaesh (2001). Robust $\mathcal{H}_{\infty}$ filtering of statioary continuous-time linear systems with stochastic uncertainties. IEEE Trans. Aut. Contr. 46, 1788-1793.

Has'minskii, R.Z. (1980). Stochastic Stability of Differential Equations. Siijthoff and Noordhoff. Aplhen aan den Rijn, The Netherlands.
Hinrichsen, D. and A.J. Pritchard (1998). Stochastic $\mathcal{H}_{\infty}$. SIAM J. Contr. Opt. 36, 1504-1538.

Kozin, F. (1969). A survey of stability of stochastic systems. Automatica 5, 95-112.

Mao, X. (1997). Exponential Stability of Stochastic Differential Equations. Horwood. London.

Ryashko, L. and H. Schurtz (1996). Mean square stability analysis of some linear stochastic systems. In: Preprints. Wierstrass Institute for Applied Analysis and Stochastics, Berlin, Germany.

Stoica, Adrian (2002). $\mathcal{H}_{\infty}$ filtering of signals subjected to multiplicative white noise. In: Proc. Triennal IFAC World Congress. Barcelona, Spain.

$\mathrm{Xu}$, S. and T. Chen (2002). Reduced-order $\mathcal{H}_{\infty}$ filtering for stochastic systems. IEEE Trans. Sign. Proc. 50, 2998-3007.

$\mathrm{Xu}$, S. and T. Chen (2003). Robust $\mathcal{H}_{\infty}$ filtering for uncertain impulsive stochastic systems under sampled measurements. Automatica 39, 500-516. 